DRAFT VERSION JULY 31, 2020

Preprint typeset using LTEX style emulateapj v. 12/16/11

\title{
DUST-SCATTERING HALO AND GIANT HARD X-RAY FLARE FROM THE SUPERGIANT FAST X-RAY TRANSIENT IGR J16479-4514 INVESTIGATED WITH XMM-NEWTON AND INTEGRAL
}

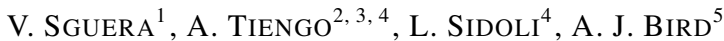 \\ Draft version July 31, 2020
}

\begin{abstract}
We report results from the analysis of XMM-Newton and INTEGRAL data of IGR J16479-4514. The unpublished XMM-Newton observation, performed in 2012, occurred during the source eclipse. No point-like X-ray emission was detected from the source, conversely extended X-ray emission was clearly detected up to a size distance compatible with a dust scattering halo produced by the source X-ray emission before being eclipsed by its companion donor star. The diffuse emission of the dust-scattering halo could be observed without any contamination from the central point X-ray source, compared to a previous XMM-Newton observation published in 2008. Our comprehensive analysis of the 2012 unpublished spectrum of the diffuse emission as well as of the 2008 re-analysed spectra extracted from three adjacent time intervals and different extraction regions (optimized for point-like and extended emission) allowed us to clearly disentangle the scattering halo spectrum from the residual point-like emission during the 2008 eclipse. Moreover, the point-like emission detected in 2008 could be separated into two components attributed to the direct emission from the source and to scattering in the stellar wind, respectively. From archival unpublished INTEGRAL data, we identified a very strong $\left(\sim 3 \times 10^{-8} \mathrm{erg} \mathrm{cm}^{-2} \mathrm{~s}^{-1}\right)$ and fast ( $\sim 25$ minutes duration) flare which was classified as giant hard X-ray flare since the measured peak-luminosity is $\sim 7 \times 10^{37} \mathrm{erg} \mathrm{s}^{-1}$. Giant X-ray flares from SFXTs are very rare, to date only one has been reported from a different source. We propose a physical scenario to explain the origin in the case of IGR J16479-4514.
\end{abstract}

Subject headings: accretion - High mass X-ray binary stars - X-ray transient sources

\section{INTRODUCTION}

One of the major outcomes of the INTEGRAL mission, launched in 2002, has been the discovery of a new class of Supergiant High-Mass X-ray Binaries (SGXBs) during systematic scans of the Galactic plane: the Supergiant Fast X-ray Transients (SFXTs, Sguera et al. 2005, 2006). They usually host a neutron star orbiting around an early type supergiant star (Negueruela et al. 2006). In the X-ray band, they show a rather well defined set of peculiar characteristics (see Sidoli 2017 for a recent review) which were never seen from previously known classical SGXBs: i) bright $\left(\sim 10^{36} \mathrm{erg} \mathrm{s}^{-1}\right)$ and fast (few hours to few days) X-ray transient behaviour ii) high dynamic ranges of $10^{3}-10^{5}$ iii) low duty cycles of $(0.1-5) \%$ when observed above $20 \mathrm{keV}$.

IGR J16479-4514 is one of the very first discovered SFXTs. It was newly discovered by INTEGRAL in 2003 as an unidentified X-ray transient with no constrained duration (Molkov et al. 2003), and further in-depth studies unveiled its peculiar fast X-ray transient nature (Sguera et al. 2005, 2006). Subsequent near infrared spectroscopy allowed the identification of its optical counterpart as a supergiant star with poorly constrained distance (Chaty et al. 2008, Nespoli et al. 2008). Coley et al. (2015) have recently reported the

\footnotetext{
${ }^{1}$ INAF-OAS, Osservatorio di Astrofisica e Scienza dello Spazio, Area della Ricerca del CNR, via Gobetti 101, I-1-40129 Bologna, Italy; vito.sguera@.inaf.it

${ }^{2}$ Scuola Universitaria Superiore IUSS Pavia, piazza della Vittoria 15 , 27100 Pavia, Italy

${ }^{3}$ Istituto Nazionale di Fisica Nucleare (INFN), Sezione di Pavia, via A. Bassi 6, 27100 Pavia, Italy

${ }^{4}$ INAF-IASF, Istituto di Astrofisica Spaziale e Fisica Cosmica, Via A. Corti 12, I-20133 Milano, Italy

${ }^{5}$ School of Physics and Astronomy, University of Southampton, University Road, Southampton, SO17 1BJ, UK
}

best constrained spectral type (O7 and earlier) and distance (in the range 4.4-4.6 kpc) for the companion donor. The Xray behaviour of the source has been investigated with different satellites, e.g. INTEGRAL (Sguera et al. 2008), Swift (Romano et al. 2008, Bozzo et al. 2009), Suzaku (Sidoli et al. 2013). IGR J16479-4514 is the SFXT with the shortest known orbital period to date ( $\sim 3.3$ days, Jain et al. 2009$)$, interestingly a superorbital period of $\sim 11.88$ days has been detected as well from Swift and INTEGRAL observations (Corbet et al. 2013, Drave et al. 2013). The source duty cycle value is one of the highest among the entire sample of firm SFXT ( 3.3\%, Sidoli \& Paizis 2018). IGR J16479-4514 is known to display X-ray eclipses as discovered during an $X M M-N e w t o n$ observation which covered part of the eclipse ingress along with part of its total phase (Bozzo et a. 2008).

Here we report new results on IGR J16479-4514 as obtained from an unpublished targeted XMM-Newton observation and from archival INTEGRAL data.

\section{DATA ANALYSIS}

Throughout the paper, spectral analysis was performed using XSPEC version 12.9.0, with the photoelectric absorption model based on the Balucinska-Church \& McCammon (1992) cross-sections and Anders \& Grevesse (1989) solar abundances. To properly use the $\chi^{2}$ statistics in model fitting, all the spectra were grouped to a minimum of 30 counts per energy bin. Unless stated otherwise, errors are quoted at the 90 per cent confidence level for one single parameter of interest.

\subsection{INTEGRAL}

For our study, we used data collected with the ISGRI detector (Lebrun et al. 2003) which is the lower energy layer of the IBIS coded mask telescope (Ubertini et al. 2003) onboard INTEGRAL (Winkler et al. 2003). 
The IBIS/ISGRI public data archive (from revolution 30 to 1500 , i.e. from approximately January 2003 to January 2015) has been specifically searched for very powerful hard X-ray flares from IGRJ16479-4514 detected at Science Window level (ScWs, $\sim 2,000$ seconds duration). In particular, the data set consists of 9,578 ScWs where IGR J16479-4514 was within the total instrument field of view (FoV) of $29^{\circ} \times 29^{\circ}$ (down to zero response), i.e. regardless of its off-axis angle. For the sake of completeness, we note that a $12^{\circ}$ limit is generally applied because the response of IBIS/ISGRI is not well modelled at large off-axis values and this may introduce a systematic error in the measurement of the source fluxes. However our specific aim is to search for exceptionally powerful outbursts (i.e. flux greater than at least $500 \mathrm{mCrab}$ as explained later in the text) which are, likely, very rare events. To increase the probability of finding such events, we deliberately did not place any requirement on the off-axis angle of the source: the larger the considered FoV, the greater the chance of serendipitously detecting a powerful random flare. Because the considered flares are exceptionally bright, their eventual detection and flux would be confidently determined even when their position is in the partially coded region of the FoV. IBIS/ISGRI flux maps for each ScW were generated in the $18-60 \mathrm{keV}$ band using the offline scientific analysis software OSA 10.2. Count rates at the position of the source were extracted from individual $\mathrm{ScW}$ flux maps. This approach is particularly efficient in unveiling fast transient flares lasting only a very few hours, since the search occurs on the same timescale as the outbursts themselves. In order to specifically search for the most powerful flares, we adopted a conservative source count rate threshold of $80 \mathrm{cts} / \mathrm{s}(18-60 \mathrm{keV})$ as measured in the single $\mathrm{ScW}$ containing the peak of the flare; it translates into a $18-60 \mathrm{keV}$ flux of $\sim 500 \mathrm{mCrab}$ by assuming a Crab-like energy spectrum with photon index equal to 2.1. Throughout the paper, the conversion from the source count rate to mCrab flux has been obtained by using the most recent Crab observations at the time of writing (revolution 1856 , August 2017), i.e. $1 \mathrm{Crab}=158.13 \mathrm{cts} / \mathrm{s}(18-60 \mathrm{keV})$. The search was initially performed in the energy band 18-60 $\mathrm{keV}$, then when a candidate fast flare was found we have also checked the detection in different energy ranges (i.e. 17-30 $\mathrm{keV}, 20-40 \mathrm{keV}$ ) in order to maximize the source best significance detection. After finding a flare, we performed a more detailed timing and spectral analysis. For the latter, we used the standard 13 energy channel response matrix available at the INTEGRAL Science Data Centre (ISDC).

In addition to the investigation of the entire public archive, we have also specifically analyzed an archival targeted INTEGRAL observation of IGR J16479-4514 (revolution 1203, August 2012), not yet published in other works, which was simultaneous to an unpublished XMM-Newton observation whose results are reported for the first time in section 3.1.

\subsection{XMM-Newton}

The XMM-Newton Observatory (Jansen et al. 2001) carries three $1500 \mathrm{~cm}^{2} \mathrm{X}$-ray telescopes, each with an European Photon Imaging Camera (EPIC; $0.2-12 \mathrm{keV}$ ) at the focus. One of the EPIC uses a pn CCD (Struder et al. 2001) while the other two are equipped with MOS CCDs (Turner et al. 2001).

We discuss here two observations, one performed in 2012 (never reported in the literature), and another one done in 2008 (Bozzo et al. 2008), which we have re-analysed here for comparison to obtain a more complete view of the phenomenology during the $\mathrm{X}$-ray eclipse.

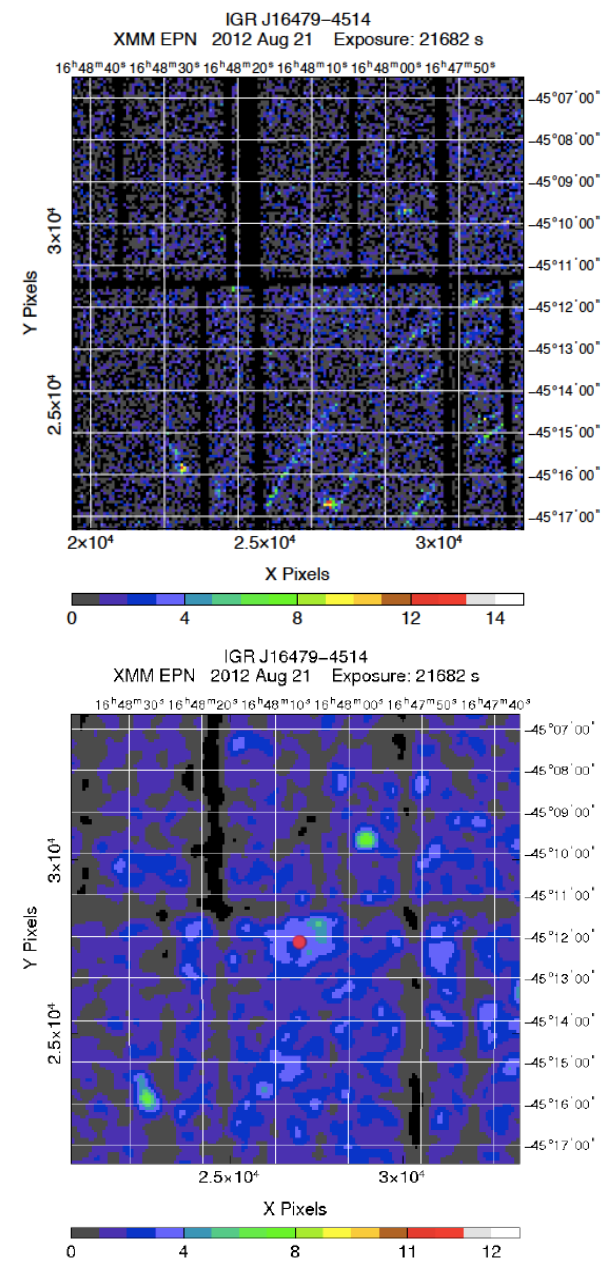

FIG. 1.- IGR J16479-4514 sky position observed by XMM-Newton/EPIC $\mathrm{pn}$, in the energy ranges $0.5-10 \mathrm{keV}$ (top panel, close-up view around the target position) and 1-3 keV (bottom panel, where the image was smoothed with a Gaussian with a width $\sigma=2$ pixels). The small red circle marks the IGR J16479-4514 infrared position (2MASS).

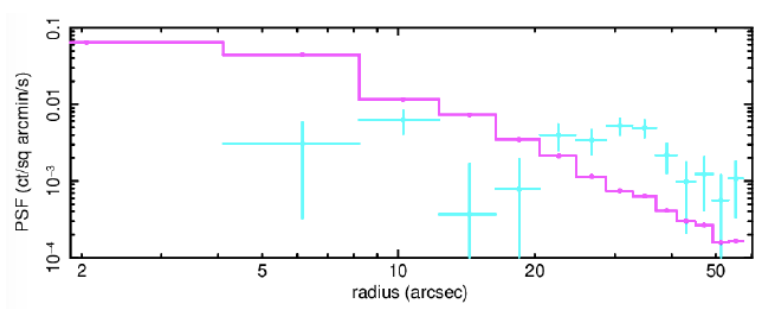

FIG. 2.- Surface brightness profiles of the $\mathrm{X}$-ray emission within $1^{\prime}$ from the source 2MASS position, in the energy range $1-3 \mathrm{keV}$, compared with the EPIC pn point spread function in the same energy range (solid line).

IGR J16479-4514 was observed with XMM-Newton on 2012 August 21 (from 11:31:03 to 18:04:47, UTC) for a net exposure of $\sim 23.9$ ks during an observation simultaneous to part of the above mentioned INTEGRAL observation. The particle background during the XMM-Newton observation was very stable. All the three EPIC cameras operated in Large Window mode and adopted the medium filter. Data were reprocessed using version 16.0.0 of the Science Analysis Software (SAS) with standard procedures and the appropriate calibration files. The response and ancillary matrices were gen- 
erated using the SAS metatask rmfgen and arfgen. Event patterns 0-4 were used when extracting EPIC pn products, and patterns $0-12$ for both the MOS cameras.

The XMM-Newton exposure was affected by stray light contamination produced by a bright source outside the field of view, as is clearly evident in Fig. 1 (top panel). The contaminating source is the LMXB GX $340+0$, which is $\sim 35$ arcmin from IGR J16479-4514. Since GX 340+0 is highly absorbed, its contribution at low energies is negligible (Fig. 11, bottom panel).

The source was observed also in 2008, from March 21 14:40 to March 22 01:30, for a net exposure time of about $30 \mathrm{ks}$, with EPIC pn in Small Window mode and the MOS in Full Frame. The MOS observations suffered from stray light contamination also during this observation.

\section{RESULTS}

\subsection{XMM-Newton}

The 2012 XMM-Newton observation was also performed during the source eclipse, according to the most recent ephemeris reported by Coley et al. (2015): orbital pe$\operatorname{riod} \mathrm{P}_{\text {orb }}=3.31961$ days, mid-eclipse time $=55081.571 \mathrm{MJD}$, eclipse duration, $\Delta \phi=0.177$, eclipse at orbital phase $\phi=0.0$.

The EPIC pn exposure covers the time interval 56160.4995-56160.75047 (MJD), implying an orbital phase range $\Delta \phi=0.02-0.09$. The eclipse ingress started on $56160.15 \mathrm{MJD}, 0.35$ days before the start of the EPIC pn exposure. At odds with the XMM-Newton observation performed in 2008, which caught the source during the eclipse ingress (Bozzo et al. 2008), IGR J16479-4514 was not detected by XMM-Newton (Fig. 1): running the detection SAS metatask edetect_chain on the three EPIC cameras separately, the three final source lists did not include any point source positionally coincident with IGR J16479-4514.

Nevertheless, there is evidence for the presence of diffuse $\mathrm{X}$-ray emission, mainly at low energies, around the source position in the EPIC pn exposure (Fig. 11, net exposure of $21.7 \mathrm{ks}$ ). This finding is confirmed by the radial distribution of the X-ray emission extracted from within 1 arcmin from the 2MASS source position. We compared this profile with the distribution expected from a point-like source (Fig. 22), resulting in a clearly diffuse emission. Since soft X-rays are efficiently scattered by interstellar dust, this diffuse emission might be the X-ray halo produced by the bright X-ray emission of IGR J16479-4514 before eclipse scattered by a large number of dust grains along the line of sight. Depending on the scattering angle and the distance of the dust, due to the longer path, the scattered emission can reach the observer with a time delay up to several days (see, e.g., Pintore et al. 2017 and references therein).

\subsubsection{XMM-Newton spectroscopy}

The EPIC pn, MOS1 and MOS2 spectra of the diffuse Xray emission observed in 2012 were extracted from an annular region with inner radius of 20 arcsec and outer radius of 40 arcsec, centered at the IGR J16479-4514 position. The background for the three cameras was extracted from two rectangular regions located in the same CCD and not contaminated by stray light, with a total area of $7,200 \operatorname{arcsec}^{2}$.

To test the dust-scattering halo hypothesis and search for spectral evolution during eclipse, we compared the background-subtracted spectrum to the corresponding spectra extracted in different time intervals from the 2008 XMMNewton observation, where the presence of a dust halo was

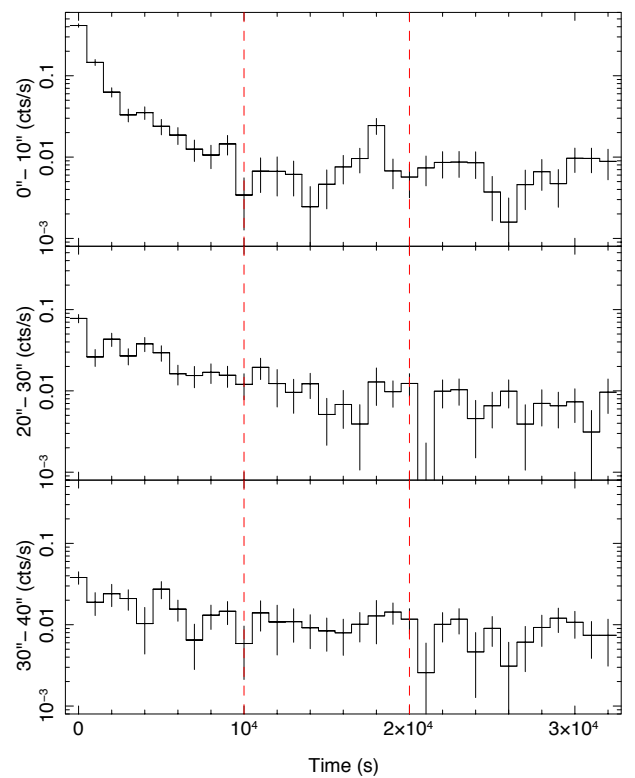

FIG. 3.- EPIC pn background-subtracted light curve of the 2008 observation extracted in the $1.5-5 \mathrm{keV}$ energy band from a circle of 10 arcsec radius (top panel) and two annuli of inner and outer radii of 20 and 30 arcsec (middle panel) and 30 and 40 arcsec (bottom panel), centered at the IGR J16479-4514 position. The bin time is $1000 \mathrm{~s}$ and the vertical lines indicate the three time intervals used for spectral analysis (interval 1,2,3 as reported in Table 1).

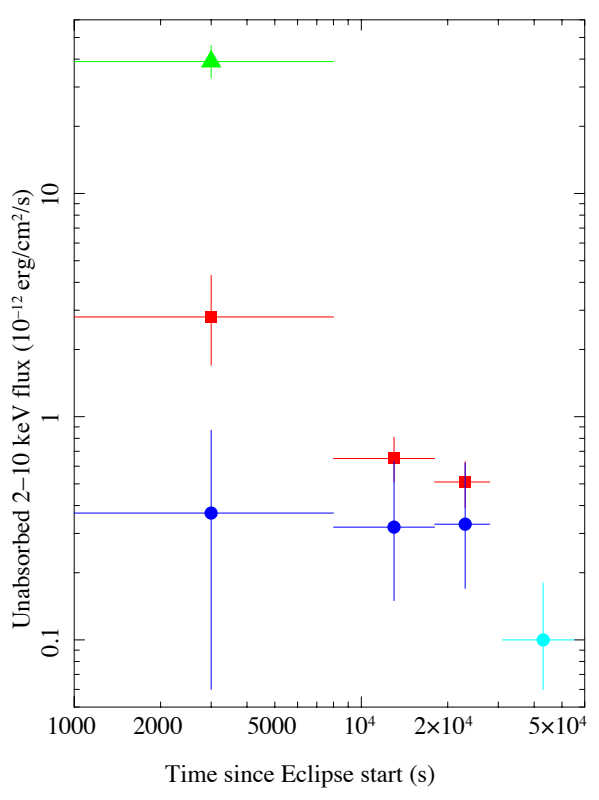

FIG. 4.- Unabsorbed 2-10 keV flux of different components (as reported in Table 11 in the 2008 (within $30 \mathrm{ks}$ since the eclipse start) and 2012 (more than $30 \mathrm{ks}$ after the eclipse start) spectra of the point source (within 10 arcsec; red squares and green triangle) and diffuse emission (from 20 to 40 arcsec; circles). The cyan circle indicates the total flux of the diffuse emission in 2012 and the blue circles are the fluxes of the halo component in the 2008 diffuse emission spectra. The green triangle is the flux of the direct component, which is significantly detected only in the interval 1 of the 2008 point source emission, whereas the red squares are the fluxes of the point source scattered component detected in the 2008 observation. 
TABLE 1

BEST-FIT PARAMETERS OF XMM-Newton SPECTRA WITH THE MODEL REPORTED IN EQUATION $1\left(\chi^{2}=164.7\right.$ FOR 176 DEGREES OF FREEDOM). INTERVAL 1, 2 AND 3 CORRESPOND TO THE PN SPECTRA OF THE TIME INTERVALS OF THE 2008 OBSERVATION, AS DEFINED IN FIG. 3 , AND OBSERVATION 2 TO THE MOS AND PN SPECTRA OF THE FULL 2012 OBSERVATION.

\begin{tabular}{|c|c|c|c|c|c|c|c|}
\hline & \multicolumn{3}{|c|}{ POINT SOURCE (10 $\operatorname{arcsec})$} & \multicolumn{4}{|c|}{ DIFFUSE EMISSION (20-40 arcsec) } \\
\hline & Interval 1 & Interval 2 & Interval 3 & Interval 1 & Interval 2 & Interval 3 & Observation 2 \\
\hline $\begin{array}{l}N_{\mathrm{H}} \\
\left(10^{22} \mathrm{~cm}^{-2}\right)\end{array}$ & $6.6_{-2.4}^{+2.5(a)}$ & $6.6^{(a)}$ & $6.6^{(a)}$ & $6.6^{(a)}$ & $6.6^{(a)}$ & $6.6^{(a)}$ & $6.6^{(a)}$ \\
\hline $\begin{array}{l}N_{\mathrm{H} 1} \\
\left(10^{22} \mathrm{~cm}^{-2}\right)\end{array}$ & $33 \pm 5^{(a)}$ & - & - & $33^{(a)}$ & - & - & - \\
\hline$\alpha$ & $1.2_{-0.1}^{+0.2(a)}$ & $1.2^{(a)}$ & $1.2^{(a)}$ & $1.2^{(a)}$ & $1.2^{(a)}$ & $1.2^{(a)}$ & - \\
\hline $\begin{array}{l}I_{1}^{(b)} \\
\left(10^{-12} \mathrm{erg} \mathrm{cm}^{-2} \mathrm{~s}^{-1}\right)\end{array}$ & $39_{-6}^{+7}$ & - & - & $3.8_{-1.2}^{+1.3}$ & - & - & - \\
\hline $\begin{array}{l}I_{2}^{(b)} \\
\left(10^{-12} \mathrm{erg} \mathrm{cm}^{-2} \mathrm{~s}^{-1}\right)\end{array}$ & $2.8_{-1.1}^{+1.5}$ & $0.65_{-0.14}^{+0.16}$ & $0.51 \pm 0.12$ & $1.2_{-0.6}^{+0.5}$ & $0.32_{-0.14}^{+0.12}$ & $0.13_{-0.12}^{+0.10}$ & - \\
\hline$\beta$ & - & - & - & $4.8_{-1.3}^{+1.5(a)}$ & $4.8^{(a)}$ & $4.8^{(a)}$ & $4.8^{(a)}$ \\
\hline $\begin{array}{l}I_{\text {halo }}^{(b)} \\
\left(10^{-12} \mathrm{erg} \mathrm{cm}^{-2} \mathrm{~s}^{-1}\right)\end{array}$ & - & - & - & $0.37_{-0.31}^{+0.50}$ & $0.32_{-0.17}^{+0.31}$ & $0.33_{-0.16}^{+0.29}$ & $0.10_{-0.04}^{+0.08}$ \\
\hline $\begin{array}{l}E_{\ln 1} \\
(\mathrm{keV})\end{array}$ & $6.36_{-0.04}^{+0.06(a)}$ & $6.36^{(a)}$ & $6.36^{(a)}$ & - & - & - & - \\
\hline $\begin{array}{l}I_{\ln 1}^{(c)} \\
\left(10^{-6} \text { photons } \mathrm{cm}^{-2} \mathrm{~s}^{-1}\right)\end{array}$ & $10 \pm 3^{(a)}$ & $10^{(a)}$ & $10^{(a)}$ & - & - & - & - \\
\hline $\begin{array}{l}E_{\ln 2} \\
(\mathrm{keV})\end{array}$ & $6.94 \pm 0.09^{(a)}$ & $6.94^{(a)}$ & $6.94^{(a)}$ & - & - & - & - \\
\hline $\begin{array}{l}I_{\ln 2}^{(c)} \\
\left(10^{-6} \text { photons } \mathrm{cm}^{-2} \mathrm{~s}^{-1}\right)\end{array}$ & $8 \pm 3^{(a)}$ & $8^{(a)}$ & $8^{(a)}$ & - & - & - & - \\
\hline
\end{tabular}

\footnotetext{
(a) Linked parameters

(b) Unabsorbed flux in the $2-10 \mathrm{keV}$ energy band.

(c) Total photon flux in the line.
}

already suggested (Bozzo et al. 2008). In this case, only pn data can be used because the stray light from GX $340+0$ in the MOS cameras reached the region where IGR J16479-4514 is located. That observation started at the beginning of the eclipse and Bozzo et al. (2008) performed a time-resolved spectral analysis by dividing the observation into two time intervals, corresponding to the initial $4 \mathrm{ks}$ of high, rapidly decaying flux and the remaining $28 \mathrm{ks}$ of low, almost constant, flux. To better separate the direct and scattered components, that are expected to have a different spatial distribution and temporal evolution, we extracted and analysed spectra from three time intervals (see Fig. 3), sampling also the possible evolution during the low flux state, and from two different extraction regions: a 10 arcsec circle to maximize the contribution from the central point source and the same annulus used for the 2012 spectrum to study the evolution of the possible dust-scattering halo.

Similarly to Bozzo et al. (2008) we adopt a spectral model consisting of three power-law, two Gaussian and two photoelectric absorption components, combined in this way:

$$
\begin{aligned}
I(E)= & e^{\sigma(E) N_{\mathrm{H}}}\left[e^{\sigma(E) N_{\mathrm{H} 1}} I_{1} E^{-\alpha}+I_{2} E^{-\alpha}+\right. \\
& I_{\text {halo }} E^{-\beta}+I_{\ln 1} e^{-\left(E-E_{\ln 1}\right)^{2} /\left(2 \sigma_{\ln 1}^{2}\right)}+ \\
& \left.I_{\ln 2} e^{-\left(E-E_{\ln 2}\right)^{2} /\left(2 \sigma_{\ln 2}^{2}\right)}\right]
\end{aligned}
$$

We use this model to simultaneously fit the nine available spectra (extracted both from a 10 arcsec circle and a 20-40 arcsec annulus in the three time intervals of the 2008 observa- tion, only from a 20-40 arcsec annulus in the 2012 observation), linking together several parameters and forcing to a null normalization all the spectral components not significantly required to model each spectrum. The width of the two emission lines was fixed to the instrumental spectral resolution. The resulting best-fit parameters are reported in Table 1 .

The spectral lines were significantly detected, with consistent parameters, only in the point-source emission. On the other hand, the steepest power-law component, that can be interpreted as the contribution from the dust scattering halo, was required only in the fit of the spectra of the diffuse component. The other two power-law components have the same slope, but one of them is detected only during the first time interval and requires an additional photoelectric absorption component. These two components can be attributed to direct and small-scale scattered emission from the point source, but, due to the relatively coarse PSF of XMM-Newton mirrors, during the 2008 observation they heavily contaminate the diffuse emission extracted from $>20$ arcsec. The normalizations of the three power-law components as a function of time since eclipse start are shown in Fig. 4.

\subsection{INTEGRAL}

\subsubsection{Archival search for powerful flares}

Our search found a very powerful fast hard X-ray flare from IGR J16479-4514. Here we report on the collected results. It was detected with a significance of $\sim 9 \sigma(18-60 \mathrm{keV})$ during only one $\mathrm{ScW}$ (n. 43) in revolution 663, at MJD 54544.96. Notably the source off axis angle was $\sim 17^{\circ} .5$. The measured 
average $18-60 \mathrm{keV}$ flux was $630 \pm 70 \mathrm{mCrab}$ or $\sim 8.2 \times 10^{-9}$ $\operatorname{erg~} \mathrm{cm}^{-2} \mathrm{~s}^{-1}$. No detection was obtained in the higher energy band 60-100 keV. The ScW spanned a time range from 2008, March 19 22:37 (UTC) to March 19 23:30 (UTC).

Figure 5 shows the 18-60 keV IBIS/ISGRI source light curve extracted from the entire $\mathrm{ScW}$ with a bin time of 50 s. A single flare is very evident, being characterized by a duration of $\sim 25$ minutes as well as a remarkable peak count rate of $\sim 330 \mathrm{cts} / \mathrm{s}$ which translates into a flux of $2.10 \pm 0.26$ $\mathrm{Crab}$ (or $\sim 2.7 \times 10^{-8} \mathrm{erg} \mathrm{cm}^{-2} \mathrm{~s}^{-1}$ ). From the light curve we note that the duration of the flare is a bit shorter than the entire duration of the ScW. Bearing this in mind, we performed an imaging analysis with the good time interval (GTI) by considering only the time interval of the transient activity (i.e. from $\sim 2,700 \mathrm{~s}$ to $\sim 4,300 \mathrm{~s}$ on $\mathrm{x}$-axis in Fig. 5). By doing so, the source was significantly detected at $13.3 \sigma$ level $(18-60 \mathrm{keV})$ despite being located at large off axis angle, as it can be seen in the significance image in Fig. 6.

Unfortunately, the source was out of the IBIS/ISGRI FoV in observations performed immediately before and after the $\mathrm{ScW}$ containing the detection of the flare, and thus it is not possible to investigate with IBIS/ISGRI if further flares took place or not. To this aim, the BAT hard X-ray transient monitor on board Swift is particularly suited since it provides a much more continuous coverage thanks to its very large FoV. We retrieved the 15-50 keV Swift/BAT light curve averaged on orbital time-scale ( $\sim 96$ minutes) from the publicly available BAT monitor web page (Krimm et al. 2013). As shown in Fig. 7, no additional X-ray activity has been detected immediately before and after the occurrence of the flare. The shadowed area indicates the flaring activity detected by IBIS/ISGRI during the single ScW. We remark that both INTEGRAL/IBIS and Swift/BAT provides complementary information: i) the continuous coverage by BAT allows us to exclude the occurrence of further X-ray activity before and after the flare, ii) the finer IBIS/ISGRI light curve (50 s bin time), with respect to the BAT light curve (95 minutes bin time), allows to unambiguously catch and estimate its exact peak-time and peak-flux, respectively.

Fig. 8 shows the IBIS/ISGRI light curve of the source (18$60 \mathrm{keV}$ ) phase-folded on the orbital period. It is clearly modulated by the deep X-ray eclipse (yellow shadowed area in Fig. 8) which is consistent with the compact object being totally eclipsed by the supergiant companion. According to the most recent and precise ephemerides of Coley et al. (2015), i.e. epoch of the mid-eclipse MJD 55081.57 as $\phi=0$, then the powerful X-ray flare detected by INTEGRAL took place at orbital phase $\phi \sim 0.65$ (grey thick line in Fig. 8).

We searched for possible periodicities in the IBIS/ISGRI data of the bright flare, which eventually could be interpreted as the possible pulse period of a neutron star compac object. A very fine bin time $(0.1 \mathrm{~s})$ ISGRI light curve $(18-60 \mathrm{keV})$ was extracted from the flare using the ii-light tool in OSA 10.2 , and Solar system barycenter correction was applied to the photon arrival times. Periodicities were searched in the frequency range from $0.000628 \mathrm{~Hz}(\sim 1590 \mathrm{~s}$, after which the sensitivity is reduced due to the finite length of the ISGRI light curve) to $5 \mathrm{~Hz}(0.2 \mathrm{~s}$, corresponding to the Nyquist frequency of the data set). Power spectra were generated using the fast Fourier transform analysis and the Lomb-Scargle periodogram technique but no statistically significant evidence for coherent modulation was found. We estimated a $3 \sigma$ upper limit to the pulsed fraction of $\sim 5 \%(18-60 \mathrm{keV})$. Given

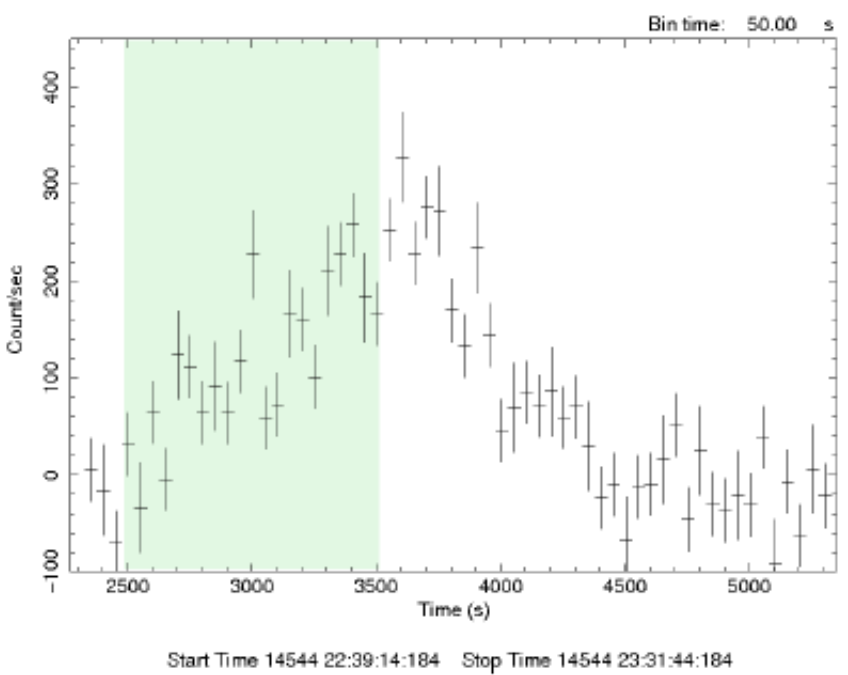

FIG. 5.- IBIS/ISGRI light curve (18-60 keV, $50 \mathrm{~s}$ bin time) of the flaring activity from IGR J16479-4514 as extracted from the single ScW n. 43 in revolution 663 . The shadowed green area indicates the portion of the flare covered by the Swift observation reported by Romano et al. (2008)

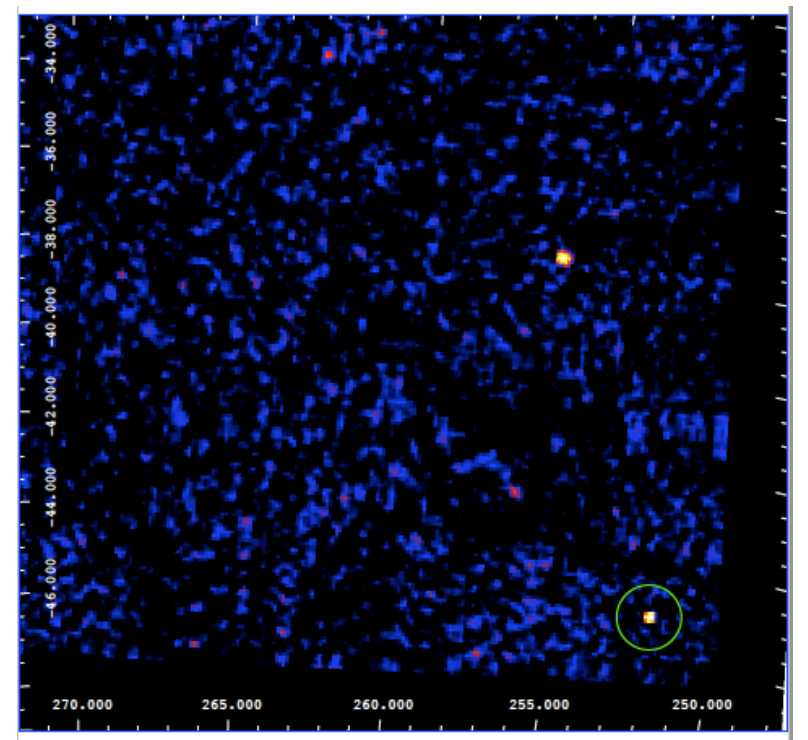

FIG. 6.- IBIS/ISGRI significance map (18-60 keV) of the single ScW n.43 in revolution 663. IGR J16479-4514 (encircled) is clearly detected at $13.3 \sigma$ level towards the edge of the FoV. The other bright source detected in the field is the HMXB $4 \mathrm{U} 1700-377$

the exceptionally high flux of the source, we have also performed a timing analysis without the image deconvolution, in a non binning way (i.e. starting from the single events). This method is particularly suited to search for very fast pulsations, e.g. up to few milliseconds. To optimize the search, we selected events according to the Pixel Illuminated Factor (PIF) which is the fractional area of each pixel exposed to the source. In particular, only photons from pixels fully illuminated by the source $(\mathrm{PIF}=1)$ were considered. By applying such PIF filter, it is possible to reduce the background and so to increase the signal to noise ratio. After barycentric correction of the photon arrival times in the original event lists, we performed both Lomb-Scargle and fast Fourier transform analysis searching for periodicities as above, however no significant evidence for a peak in the power spectra was found. 


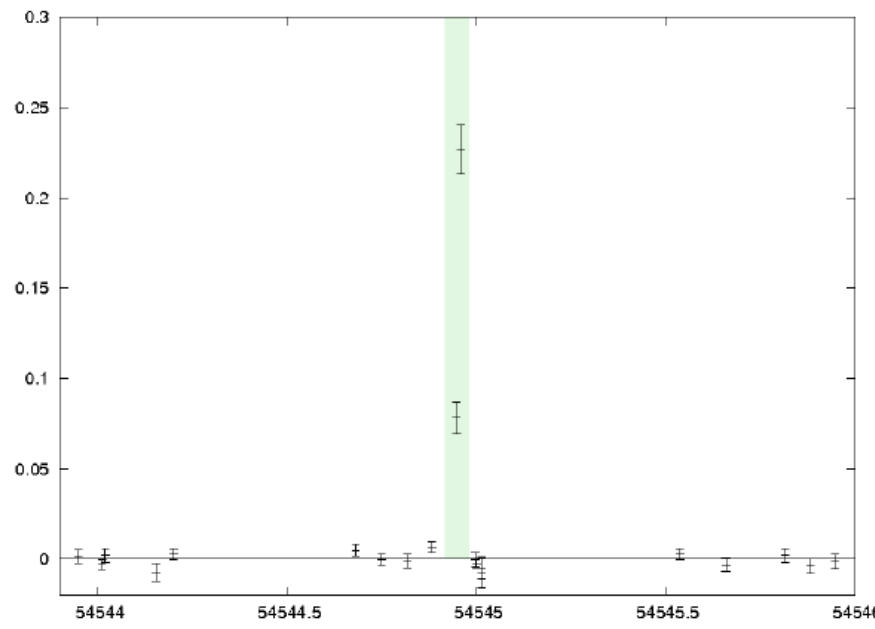

FIG. 7.- Swift/BAT light curve (15-50 keV) on orbital time-scale of the flare from IGR J16479-4514. The shadowed green area indicates the portion of the flaring activity covered by the IBIS/ISGRI observation during the single ScW n. 43. Time on the X-axis is in MJD unit.

\subsubsection{Targeted observation}

INTEGRAL performed a targeted observation of IGR J16479-4514 during revolution 1203, from 2012 August 21 04:30:00 UTC to 21 August 21:25:42 UTC. The source was in the fully coded FoV of IBIS/ISGRI for a total effective on-source exposure of $\sim 16$ ks. As shown in Fig. 8 (where the orbital phases corresponding to the start and end time of the INTEGRAL observation are indicated by means of two blue lines) almost the entire observation took place during the eclipse, when the compact object is totally eclipsed by the supergiant companion donor.

The IBIS/ISGRI mosaic was made in the energy band 22$60 \mathrm{keV}$ (to take into account the evolution of the IBIS/ISGRI energy threshold that occurred from revolution number 900 on). The source was not detected in the mosaic, nor in any single $\mathrm{ScW}$. As a result, we inferred a $22-60 \mathrm{keV} 3 \sigma$ upper limits of $4.6 \mathrm{mCrab}\left(\sim 5 \times 10^{-11} \mathrm{erg} \mathrm{cm}^{-2} \mathrm{~s}^{-1}\right)$. For comparison, we note that the lowest out-of-outburst as well as out-ofeclipse hard X-ray emission detected by IBIS/ISGRI is known to be of the order of $\sim 1.7 \times 10^{-11} \mathrm{erg} \mathrm{cm}^{-2} \mathrm{~s}^{-1}(20-60 \mathrm{keV}$, Sguera et al. 2008, Walter \& Zurita 2007), i.e. only a factor of 3 lower than this upper limit during the eclipse.

\section{DISCUSSION}

\subsection{X-ray eclipse}

During the XMM-Newton observation performed in 2012, no point-like X-ray emission was detected from IGR J16479-4514. However, extended X-ray emission was clearly observed up to a distance of at least 40 arcsec. As already noted by Bozzo et al. (2008), such a size is compatible with a dust scattering halo produced by the X-ray emission of IGR J16479-4514 before being eclipsed by its companion. Also its spectrum, significantly softer than the typical spectrum of this X-ray source out of eclipse, is consistent with that expected from dust-scattering, whose efficiency steeply decreases with photon energy (see, e.g., Figure 6 in Draine 2003).

From a time-resolved spectral analysis of the 2008 observation, performed immediately after a bright flare and during earlier phases of the eclipse, we could clearly disentangle

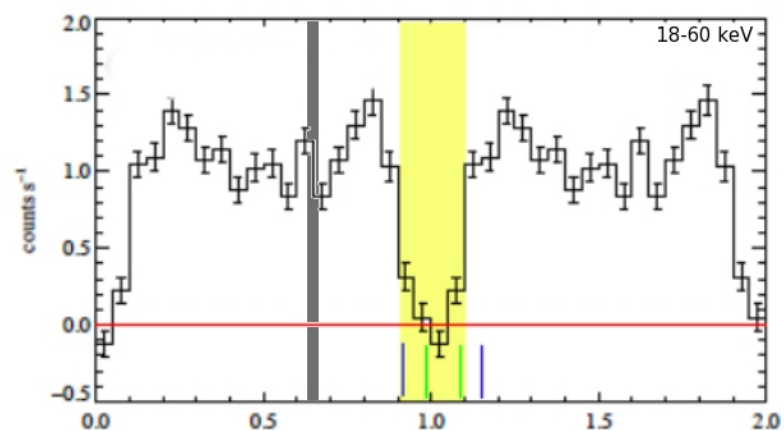

FIG. 8.- IBIS/ISGRI light curve of IGR J16479-4514 (18-60 keV) phasefolded on the orbital period. Phase 0 is defined at the time of mid-eclipse MJD 55081.57 according to the most recent and precise ephemerides of Coley et al. (2015). The shadowed yellow area indicates the entire duration of the X-ray eclipse. The grey thick line marks the occurrence of the powerful flare detected by IBIS/ISGRI. The two blue lines (green lines) indicate the start time and the end time of the targeted INTEGRAL (XMM-Newton) observation.

the scattering halo component from two distinct components from the central point source: the brightest one is detected only during the initial phases of the eclipse and is characterized by a very significant excess of photoelectric absorption and can therefore be attributed to the direct emission from IGR J16479-4514 passing through a dense local environment. The second point-like component, instead, is detected throughout the full 2008 observation and declines faster than the dust scattering halo (see Fig. 4); its spectral slope is comparable to the direct component, but its absorption is much smaller and, considering also the large equivalent width of the detected emission lines, can be attributed to scattering in the stellar wind. The largest absorption of the direct component suggests the presence of an absorbing structure in the orbital plane, which does not obscure the scattered emission, emerging more clearly during eclipse. A spatially larger but much less dense structure surrounding IGR J16479-4514 might be responsible for part of the absorption affecting all the spectral components, since its value of $\mathrm{N}_{\mathrm{H}}=(6 \pm 2) \times 10^{22} \mathrm{~cm}^{-2}$ is significantly larger than the total Galactic absorption column expected in this sky direction $\left(\mathrm{N}_{\mathrm{H}} \sim 2 \times 10^{22} \mathrm{~cm}^{-2}\right.$; Willingale et al. 2013). However, we note that a similar excess is measured in two nearby bright X-ray binaries, GX 340+0 (Miller et al. 2016) and XTE J1652-453 (Hiemstra et al. 2011) and therefore its origin might be the complex structure of the interstellar medium in this Galactic region rather than the local environment of IGR J16479-4514.

The comparison of the fluxes of the different components shows how the contribution from the dust scattering halo increases at later eclipse phases (see Fig. 4). Moreover, the flux of the halo during the 2012 observation is more than 3 times smaller than during the 2008 observation, which indicates that also the flux of IGR J16479-4514 out of eclipse was significantly smaller in the hours/days preceding the most recent observation. For these reasons, in 2012 the dust scattering halo could be observed without apparent contamination from the central point source.

\subsection{Giant hard X-ray flare}

INTEGRAL detected a fast hard X-ray flare from IGR J16479-4514 ( 25 minutes duration) which reached a remarkable peak flux of $2.10 \pm 0.26$ Crab $(18-60 \mathrm{keV})$. It translates into a X-ray luminosity of $(7.0 \pm 0.9) \times 10^{37} \mathrm{erg} \mathrm{s}^{-1}$ if we assume the most constrained value of $4.6 \mathrm{kpc}$ for the dis- 
tance, to date (Coley et al. 2015). This is the most powerful flare ever detected from the source. Moreover, it is one of the most powerful flares ever detected from any SFXT, in this respect being second only to the giant soft X-ray flare detected by Swift/XRT from the SFXT IGR J17544-2619 (Romano et al. 2015), which reached a peak flux (luminosity) of $2.1 \mathrm{Crab}$ $\left(3 \times 10^{38} \mathrm{erg} \mathrm{s}^{-1}\right)$ but in the softer energy band $0.3-10 \mathrm{keV}$, so a proper comparison between the two events is not possible. Its physical origin was explained in the framework of accretion from a transient disk (Romano et al. 2015). Previously to our current work, the dynamic range of the source above $20 \mathrm{keV}$ was of the order of $\sim 350$, as reported in the literature (Sguera et al.2008) by considering the highest measured luminosity level in outburst $\left(1.5 \times 10^{37} \mathrm{erg} \mathrm{s}^{-1}\right.$ at $4.6 \mathrm{kpc}$ distance, $20-60 \mathrm{keV}$ ) and the lowest detected hard X-ray emission level outside outburst $\left(4.3 \times 10^{34} \mathrm{erg} \mathrm{s}^{-1}\right.$ at $4.6 \mathrm{kpc}$ distance, $20-60$ $\mathrm{keV}$ ). Our reported giant hard X-ray flare, the strongest ever, allows us to push further the dynamic range up to a value of $\sim 1630$.

For the sake of completeness, we note that this flare from IGR J16479-4514 has been previously studied with Swift by Romano et al. (2008). The flare triggered the BAT monitor on board Swift on 2008 March 19 at 22:44:45 UT (Barthelmy et al. 2008). Subsequently the satellite slewed to the target with the XRT instrument, however its observation temporally covered the flare only during its rising for about 1,000 seconds (see shadowed green area in Fig. 5). Romano et al. (2008) mainly performed a Swift broad band X-ray spectral study of this flare. Here we note that, because of the partial Swift temporal coverage of the flare which was limited only to its rising part, it was not possible to provide any exact measurement on its duration, peak-time and peak-flux. Conversely, our newly reported INTEGRAL detection fully covered the entire duration of the flare allowing such measurements and unveiling its very energetic nature. In this respect our reported INTEGRAL results are novel and complementary with those previously reported from the Swift observation (Romano et al. 2008).

This exceptionally energetic flare raises questions on its physical origin. Within the quasi spherical settling accretion model of Shakura et al. (2012, 2013, 2014), the production of bright X-ray flares from SFXTs (i.e. $\mathrm{L}_{x}>10^{36} \mathrm{erg} \mathrm{s}^{-1}$ ) is triggered by sporadic capture of magnetized stellar wind plasma by the magnetized compact object. The consequent magnetic reconnection increases the magnetospheric plasma entry rate, this results in abundant production of X-ray photons, strong Compton cooling and ultimately in the unstable accretion of the entire quasi-static shell previously formed above the neutron star magnetosphere. Clearly the shell can be reformed by new wind capture so the flares production can be repeated as long as the rapid mass entry rate into the magnetosphere is sustained. In this scenario, a bright flare must be produced on the free fall time scale of the shell (i.e. $\sim 1,000-10,000 \mathrm{~s}$ ) with a typical energy released of $\sim 10^{39}$ ergs, corresponding to a typical mass of the shell of $\sim 10^{19} \mathrm{~g}$. This view is broadly consistent with the typically measured energy released in known SFXTs bright flares detected by $I N$ TEGRAL (in the range $10^{38}-10^{40} \mathrm{ergs}$ ) as well as with the corresponding measured mass fallen onto the compact object $\left(10^{18}-10^{20} \mathrm{~g}\right)$ (Shakura et al. 2014). As for the specific case of IGR J16479-4514, we took into account the peak luminosity of the giant hard X-ray flare and accordingly calculated the corresponding released energy and accreted mass following Shakura et al. (2014). We derived values of $\sim 5 \times 10^{41}$ ergs and $\sim 6 \times 10^{21} \mathrm{~g}$, respectively. Clearly, this is an exceptional event with respect to the usual flares typically detected, since it released a much greater amount of energy and it required a much larger amount of accreted material than usual.

IGR J16479-4514 is known to undergo a regular flaring activity at a specific orbital phase $\phi \sim 0.65$ (if the epoch MJD 55081.57 is assumed as $\phi=0$ according to Coley et al. 2015). This interesting behaviour has been firstly reported by Bozzo et al. (2009) using Swift observations and subsequently confirmed by Sidoli et al. (2013) through a nearly complete orbital monitoring with Suzaku. This characteristic likely indicates the presence of a phase-locked large-scale structure in the supergiant wind, consecutively the flares are likely triggered by higher accretion rate onto the compact object during its passage inside of it. We note that such large-scale structures are believed to be an ubiquitous characteristic of all isolated supergiant stars (Puls et al. 2008, Massa \& Prinja 2015). In particular Corotating Interaction Regions (CIRs) have been suggested as a potential candidate (Mullan 1984, Cranmer \& Owocki 1996), being characterized by an over-density with respect to the unperturbed surrounding stellar wind. Since the giant flare detected by INTEGRAL occurred at orbital phase $\phi=0.648 \pm 0.002$ (if the epoch MJD 55081.571 is assumed as $\phi=0$ and $3.31961 \pm 0.00004$ days is assumed as orbital period according to the most recent and precise results of Coley et al. 2015), we propose that the interaction of the compact object with a CIR in the supergiant wind, during its passage inside of it, has provided the needed very large amount of accreted material.

In order to support our hypothesis, we note that Corbet \& Krimm (2013) reported the light curve of IGR J16479-4514 folded on its superorbital period of $\sim 11.88$ days (see their figure 8 ), it clearly shows a sharp peak at superorbital phase in the range $\Phi=0-0.08$ (if phase $\Phi=0$ is assumed at MJD 55996 according to Corbet \& Krimm 2013). Recently Bozzo et al. (2017) proposed that the interaction between the compact object and the CIRs in the wind of its supergiant companion could drive the superorbital periodicities observed in some SFXTs as well as SGXBs. When the NS encounters the CIR, the different velocity and density of this structure compared to the surrounding stellar wind produces the required long-term variation of the mass accretion rate to give rise to a superorbital modulation with the observed intensity. Under this hypothesis, we calculated for IGR J16479-4514 a CIR rotating period of $\sim 4.607$ days according to equation 1 in Bozzo et al. (2017), with the assumption that there is a single arm CIR in the stellar wind. However, we note that it cannot be excluded apriori the possibility of multiple n CIRs (in this case the rotation period would be $4.607 / \mathrm{n}$ ). We used the value of 4.607 days to calculate the superorbital phase corresponding to the giant hard X-ray flare detected by INTEGRAL, and we found a value of $\Phi=0.035 \pm 0.021$. Remarkably, we note that this is in agreement with the superorbital phase of the peak (in the range $\Phi=0-0.08$ ) corresponding to maximum flux in the light curve of IGR J16479-4514 folded on its superorbital period (see figure 8 in Corbet \& Krimm 2013). This supports our hypothesis that the giant hard X-ray flare has been produced likely during the passage of the compact object inside the putative CIR. As a further check, in Fig. 9 we shows the IBIS/ISGRI light curve (18-60 keV) folded on the superorbital period according to Corbet \& Krimm (2013). The used light curve covers a time range of $\sim 13$ years of $I N$ TEGRAL observations, from January 2003 to January 2015. We note that the shape of the folded INTEGRAL light curve 


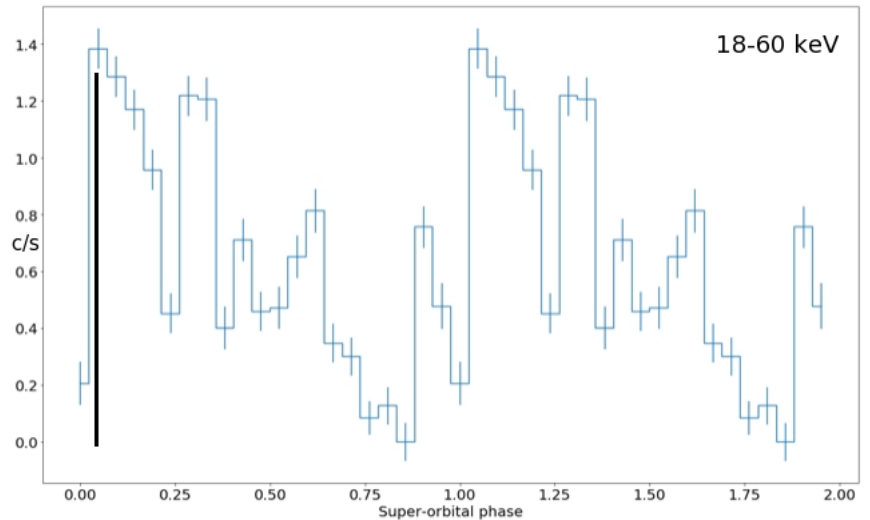

FIG. 9.- IBIS/ISGRI light curve of IGR J16479-4514 (18-60 keV) phasefolded on the superorbital period. Phase 0 is defined according to Corbet $\&$ Krimm 2013. The black line marks the occurrence of the powerful flare detected by INTEGRAL.

is broadly consistent with that of Swift/BAT as published by Corbet \& Krimm 2013 (see their Fig. 8) in a similar energy range $(15-50 \mathrm{keV})$, i.e. it is characterized by a relatively sharp rise from minimum to maximum followed by a less intensive plateau phase. However, in addition we point out that the shape of the folded INTEGRAL light curve is bumpier than that of Swift/BAT since it shows some extra peaks which are very likely due to hard X-ray flares from the source presumably detected by INTEGRAL, which on the contrary were not detected by Swift/BAT due to its higher instantaneous sensitivity, with respect to INTEGRAL, on the short time scale typical of the detected flares (i.e. 2,000 seconds).

We note that the putative arm CIR should likely be a structure stable over timescales of several years, as suggested by the detection with INTEGRAL, Swift/BAT and Suzaku of several X-ray flares at $\phi \sim 0.65$ spanning a temporal range of at least $\sim 7$ years. The stability is further corroborated by the persistent detection of the superorbital modulation in the Swift/BAT light curve covering a timescale of about 8.5 years (Corbet et al. 2013). The giant flare detected by INTEGRAL is exceptionally energetic compared to typical flares observed at the same orbital phase, this could imply changes of the mass accretion rate related to cycle-to-cycle variations of the arm CIR density (e.g. as in the case of the wind fed supergiant HMXB IGR J16493-4348, Coley et al. 2019).

\section{SUMMARY AND CONCLUSIONS}

In this work we presented new XMM-Newton and INTEGRAL results on the SFXT IGR J16479-4514. The main results and conclusions from our analysis are the following:

(i) no point source was detected during the unpublished 2012 XMM-Newton observation, performed during eclipse. This allowed us to clearly detect, without any contamination, the diffuse soft X-ray emission discovered in the previously published 2008 XMM-Newton observation during eclipse and tentatively interpreted as a dust-scattering halo (Bozzo et al. 2008). The significantly lower flux observed in the 2012 observation strongly supports the dust halo interpretation.

(ii) a time-resolved re-analysis of the archival $2008 X M M$ Newton observation, adopting more time intervals and source extraction regions (optimized either for point-like and diffuse emission) than in Bozzo et al. (2008), has allowed us to better characterize the point-like emission during eclipse, disentangling two components with different evolution timescales and power-law spectra with the same slope but significantly different photoelectric absorption.

(iii) INTEGRAL detected an exceptionally energetic (peak luminosity of $\left.\sim 7 \times 10^{37} \mathrm{erg} \mathrm{s}^{-1}, 18-60 \mathrm{keV}\right)$ and fast (25 minutes duration) giant hard X-ray flare. They are very rare from SFXTs, this would be only the second one reported in the literature after that from the SFXT IGR J17544-2619 detected in a much softer energy band (peak luminosity of $\sim 2 \times 10^{38}$ $\operatorname{erg~s}^{-1}, 0.2-10 \mathrm{keV}$ )

(iv) the detected giant hard X-ray flare required an amount of accreted material $\left(\sim 7 \times 10^{21} \mathrm{~g}\right)$ about two orders of magnitude greater than usually measured from all previous detected flares from the source. The giant flare occurred at a specific orbital phase which has been previously suggested to be linked to the presence of a stable large-scale structure in the supergiant wind. We invoke CIRs in the wind as a potential candidate and consecutively we propose that the interaction of the compact object with such CIRs, during its passage inside of it, have provided the needed very large amount of accreted material necessary to produce the exceptionally energetic hard $\mathrm{X}$-ray flare.

\section{ACKNOWLEDGMENTS}

We would like to thank the referee for valuable comments which helped us to improve the manuscript. LS thanks M. Marelli for interesting discussions. AT acknowledges funding in the framework of the project ULTraS ASI-INAF contract N. 2017-14-H.0.

\section{REFERENCES}

Anders E. \& Grevesse N. 1989, Geochimica et Cosmochimica Acta, 53, 197

Balucinska-Church, M. \& McCammon, D. 1992, ApJ, 400, 699

Barthelmy S. D., Baumgartner, W. H., Burrows, D. N., et al. 2008, GCN Circ.7466

Bozzo, E. and Stella, L. and Israel, G. and Falanga, M. and Campana, S. 2008, MNRAS, 391L, 108

Bozzo, E., Giunta, A., Stella, L., et al. 2009, A\&A, 502, 21

Bozzo, E., Oskinova, L., Lobel, A., et al. 2017, A\&A, 606L, 10

Coley, J. B., Corbet, R. H. D., Krimm, H. A., 2015, ApJ, 808, 140

Coley, J. B., Corbet, R. H. D., Furst, F., 2019, ApJ, 879, 34

Corbet, R. H. D., Krimm, H. A., 2013, ApJ, 778, 45

Chaty, S., Rahoui, F., Foellmi, C., et al. 2008, A\&A, 484, 783

Cranmer, S. R., Owocki, S. P., 1996, ApJ, 462, 469

Draine, B. T., ApJ, 598, 1026

Drave, S. P., Bird, A. J., Goossens, M. E., et al. 2013, Astron. Tel., 5131

Hiemstra, B., et al. 2011, MNRAS, 411, 137

Jain, C., Paul, B., Dutta, A, 2009, MNRAS397, L11
Jansen, F., Lumb, D., Altieri, B., et al. 2001, A\&A, 365L, 1

Krimm, H. A., Holland, S. T., Corbet, R. H. D., et al. 2013, ApJS, 209, 14

Lebrun F., et al., 2003, A\&A, 411, 141

Massa, D., Prinja, R. K., 2015, ApJ, 809, 12

Miller, J. M., et al. 2016, ApJL, 822, L18

Molkov, S., Mowlavi, N., Goldwurm, A., et al. 2003, Astron. Tel., 176 Mullan, D. J., 1984, ApJ, 283, 303

Negueruela I., Smith D., Reig P., et al. 2006, ESA SP-604: X-ray Universe 2005, Wilson A. editor, 2006, 165

Nespoli, E., Fabregat, J., Mennickent, R. E., 2008, A\&A, 486, 911

Puls, J.,Vink, J. S., Najarro, F., 2008, A\&ARv, 16, 209

Pintore, F., Tiengo, A., Mereghetti, S., et al. 2017, MNRAS, 472, 1465

Romano, P., Sidoli, L., Mangano, V., et al. 2008, ApJ, 680L, 137

Romano P., Bozzo E., Mangano V., et al. 2015, A\&A, 576L, 4

Sguera, V., Barlow, E. J., Bird, A. J., et al. 2005, A\&A, 444, 221

Sguera, V., Bazzano, A., Bird, A. J., et al. 2006, ApJ, 646, 452

Sguera, V., Bassani, L., Landi, R., et al. 2008, A\&A, 487, 619 
Shakura, N., Postnov, K., Kochetkova, A., et al. 2012, MNRAS, 420, 216 Shakura, N., Postnov, K., Hjalmarsdotter, L., 2013, MNRAS, 428, 670S

Shakura, N., Postnov, K., Sidoli, L., et al. 2014, MNRAS, 442, 2325

Sidoli, L., Esposito, P., Sguera, V., et al. 2013, MNRAS, 429, 2763

Sidoli, L., 2017, Proceedings of the XII Multifrequency Behaviour of High Energy Cosmic Sources Workshop, held on 12-17 June 2017, Palermo, Italy (PoS(MULTIF2017)052)
Sidoli \& Paizis 2018, MNRAS, 481, 2779

Struder, L., Briel, U., Dennerl, K., 2001, A\&A, 365L, 18

Turner, M. J. L., Abbey, A., Arnaud, M., et al. 2001,A\&A, 365L, 27

Ubertini P., et al., 2003, A\&A, 411, L131

Walter, R., Zurita Heras, J., et al. 2007, A\&A, 476, 335

Willingale R., et al., 2013, MNRAS, 431, 394

Winkler C., et al., 2003, A\&A, 411, L1 